\title{
Etude des composés neutres volatils formés au cours du gratinage du Gruyère
}

\author{
par \\ J. P. DUMONT. G. PRADEL, Sylviane ROGER \\ et J. ADDA \\ Station Centrale de Recherches laitières \\ et de Technologie des Produits Animaux, I.N.R.A. \\ 78350 Jouy-en-Josas (France)
}

\section{I. - INTRODUCTION}

Traditionnellement les fromages à pâte cuite du type Gruyère ou Emmental sont consommés soit comme tels ou bien sont utilisés dans de nombreuses préparations culinaires et donnent en particulier après cuisson au four (gratinage) un arôme tout à fait caractéristique aux aliments. Il nous a semblé intéressant d'essayer de voir dans un premier temps quelles étaient les substances formées au cours du gratinage en étudiant successivement les composés volatils présents dans un fromage de Comté de bonne qualité puis les substances émises au cours de l'opération de gratinage elle-même, substances qui dans la pratique culinaire normale se dégagent dans l'atmosphère et enfin les produits volatils isolés de la masse de fromage fondu qui constituent le gratin proprement dit. D'autre part, pour se rapprocher davantage des conditions de la pratique courante, cette expérience a été répétée en gratinant une même quantité de fromage sur un lit de pâtes cuites.

\section{II. - MATERIEL ET METHODES}

\section{Cuisson au four}

On utilise un four électrique ménager (Brandt E 701) modifié de façon à permettre la condensation des vapeurs qui se dégagent au cours de la cuisson dans une série de pièges qui, suivant les cas, étaient soit refroidis à l'azote liquide soit contenaient une solution $\mathrm{HCl} 2 \mathrm{~N}$ saturée en 2,4-dinitrophénylhydrazine.

Le four est préalablement chauffé à $240^{\circ} \mathrm{C}$ environ (thermostat 8) puis la cuisson est réalisée en utilisant le gril, sur un échantillon 
de $750 \mathrm{~g}$ de fromage râpé réparti au fond d'un plat pyrex vide ou contenant un poids égal de pâtes cuites.

\section{Extraction}

2.1. Les pièges où l'on a condensé les vapeurs sont rincés avec de l'éther éthylique purifié. Les acides organiques sont éliminés par lavage avec une solution alcaline puis la phase éthérée est séchée sur sulfate de sodium anhydre ; concentrée sous colonne de Vigreux elle fournit l'extrait utilisé pour l'analyse.

2.2. Le fromage ou les produits ayant subi de la cuisson (pâtes seules, gratin, gratin + pâtes) sont mis en suspension dans de l'eau distillée filtrée sur charbon actif. Cette suspension est soumise à une distillation sous reflux en utilisant le montage décrit par Forss et al. [6], modifié (fig. 1) par Ramshaw. Les produits les plus volatils se condensent avec une certaine quantité d'eau dans les pièges refroidis par de l'azote liquide.

Le contenu du ballon $\mathrm{B}$, ramené à un volume de $10 \mathrm{ml}$ par cryoconcentration est mélangé avec le contenu des pièges. Après ajustement du $\mathrm{pH}$ on extrait par l'éther éthylique. La phase éthérée est séchée sur sulfate de sodium et concentrée.

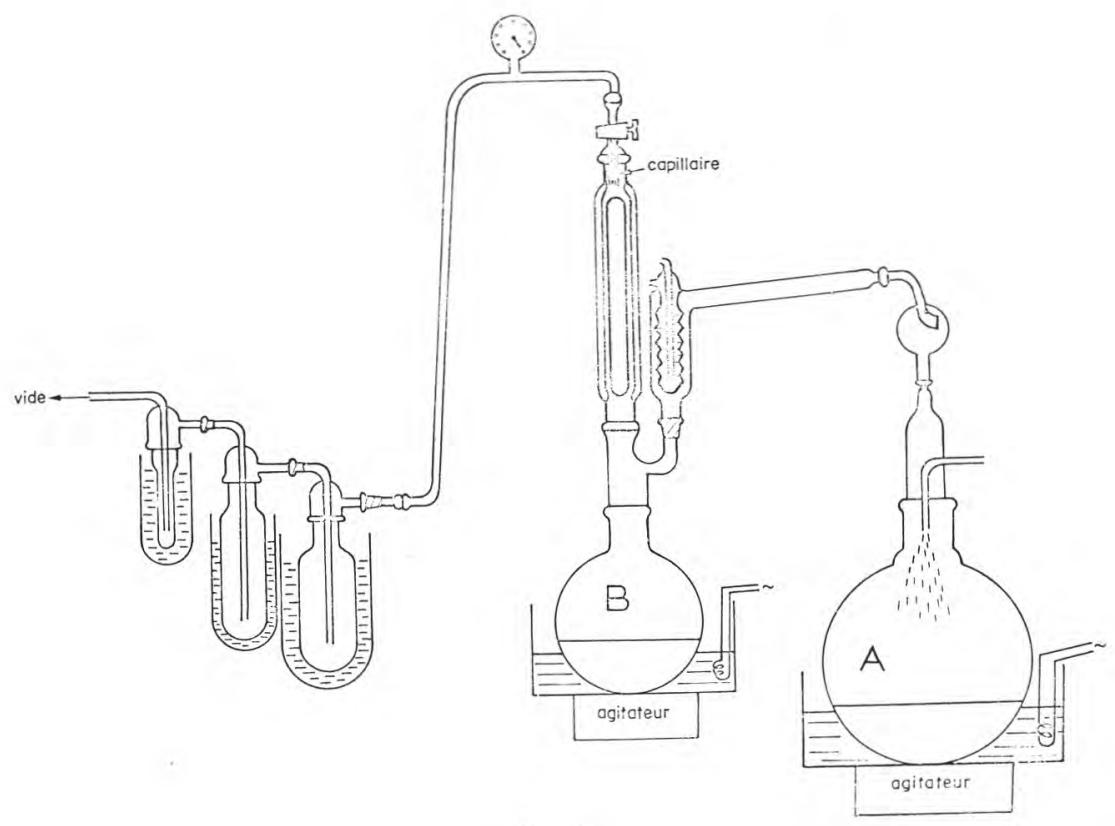

fig. 1

Dispositif de distillation sous reflux 
2.3. Les extractions par solvant introduisent des artefacts et la concentration de l'extrait conduit à la perte des substances les plus volatiles c'est pourquoi il a semblé intéressant d'utiliser la méthode d'extraction décrite par Jennings [7] où un " head-space " est piégé sur une colonne de Porapak Q 80/100 mesh puis désorbé par un courant d'azote à température élevée. Les produits désorbés sont recueillis dans un capillaire en verre refroidi par de la neige carbonique.

\section{Séparation chromatographique}

3.1. Les extraits sont analysés par chromatographie en phase gazeuse. On utilise un appareil Girdel 3000 équipé d'un détecteur à ionisation de flamme, et une colonne en acier inoxydable de $100 \mathrm{~m}$ et de $1 \mathrm{~mm}$ de diamètre intérieur revêtue de silicone SF 96-Igepal CO 880 95:5. Après une période isotherme à $30^{\circ} \mathrm{C}$, la température est programmée de $30^{\circ} \mathrm{C}$ à $130^{\circ} \mathrm{C}$ à raison de $1,5^{\circ} \mathrm{C} / \mathrm{mn}$.

3.2. Identification. Elle a été réalisée à l'aide du couplage chromatographie en phase gazeuse (Girdel 3000) spectromètre de masse à faible résolution (AEI MS 20 organique) en utilisant les mêmes conditions chromatographiques que précédemment.

La confirmation de l'identité des composés mis en évidence lors de l'analyse de l'extrait total a été obtenue en fractionnant cet extrait soit par chromatographie préparative sur une phase polaire (colonne $1 / 8$ de $3 \mathrm{~m}$ de long Carbowax 20 M TPA 5 p. 100 sur chromosorb GAW DMCS 60/80), soit par chromatographie sur colonne d'acide silicique suivant la technique décrite par Palmer [13]. Chacune des fractions ainsi obtenues est réinjectée dans la colonne capillaire couplée au spectromètre de masse précédemment utilisée.

\section{Chromatographie en couche mince}

Les 2,4-DNPHones obtenues par barbottage des vapeurs émises au cours de la cuisson, dans une solution de 2,4 DNPH ont été séparées par chromatographie en couche mince. Les dérivés aliphatiques sont tout d'abord séparés des dérivés aromatiques et dicarbonyles [4], puis les dérivés aliphatiques sont séparés en sous-groupes suivant leur longueur de chaîne [1]. Chaque sous-groupe est ensuite fractionné en classes qui sont enfin reprises pour être séparées en homologues [1].

\section{Etude des composés soufrés}

Les composés soufrés ont été dosés d'une part par la méthode colorimétrique décrite par Mc Lay [11] sur un échantillon de $100 \mathrm{~g}$, d'autre part une étude chromatographique a été effectuée en utilisant la méthode mise au point par Quist et Von Sydow [14]. 


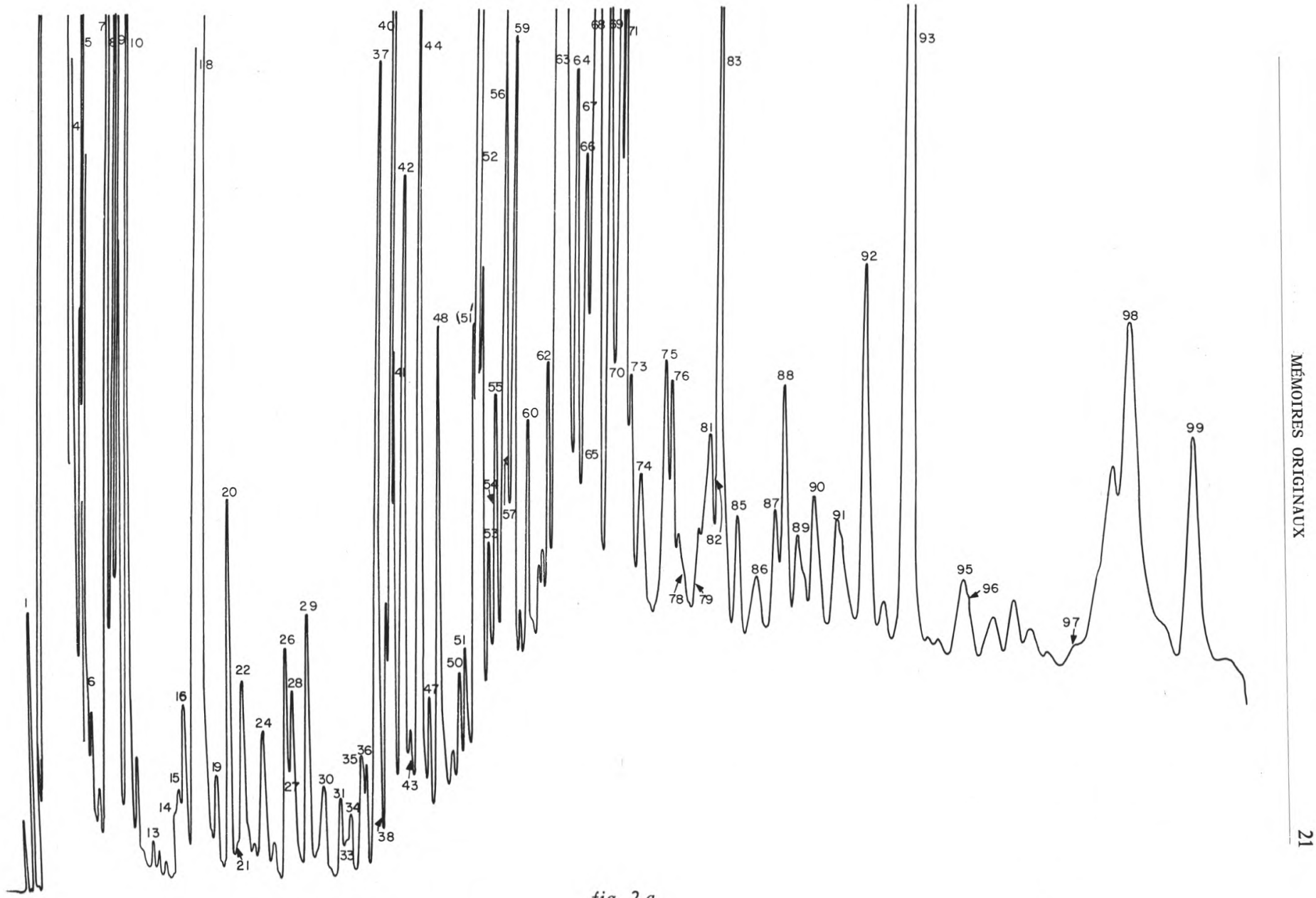




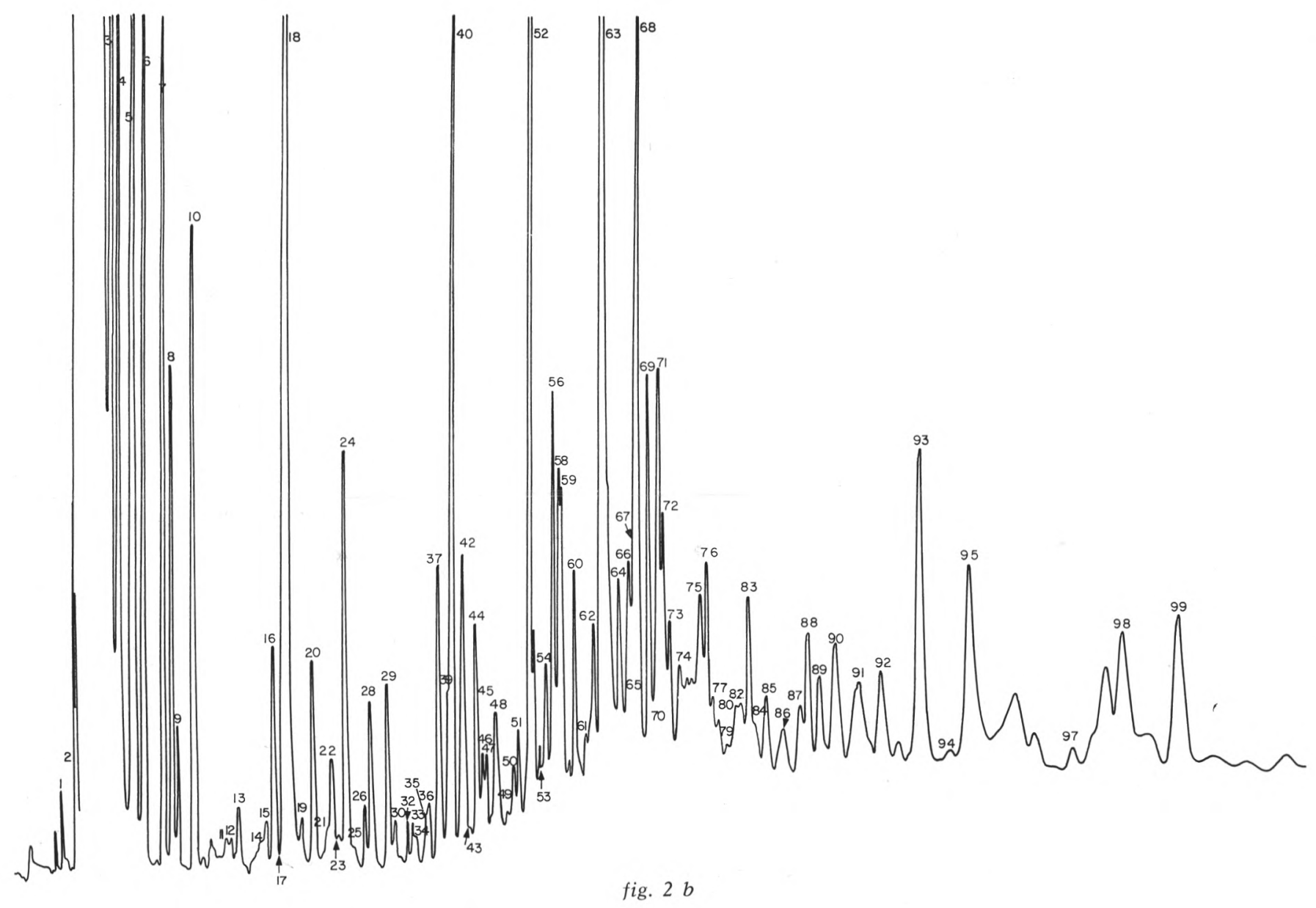




\section{fig. $2 a$ et $b$}

(a) Profil obtenu après gratinage du fromage seul (G).

(b) Profil obtenu après gratinage du fromage et des pâtes (GP). Colonne : $100 \mathrm{~m}$. $1 \mathrm{~mm}$ d.i. SF $96-$ Igepal $95: 530 \rightarrow 130^{\circ} \mathrm{C} 1,5^{\circ} \mathrm{C} / \mathrm{mn}$. 1. éthanal, 2. méthanol, 3. 2-méthylpropanal, 4. 2-butanone + propanol I + 2,3-diméthylbutane + 2-méthylpentane, 5 . hexane + acétate d'éthyle +2 -butanol, 6 . chloroforme, 7 . méthylbutanal, 8. méthylbutanal, 9. benzène, 10. n-butanol + 2-pentanone, 11. trichloroéthylène (GP), 12. éthyl furane (GP), 13. 1-pentanol + éthylpentane, 14. acétoïne, 15. 4-méthyl-2-pentanone + méthyl-2-buten-2-al-1, 16. diméthyldisulfure, 17. 3-méthyl-2pentanone (GP), 18. 3-méthyl-1-butanol, 19. 2-méthyl-1-butanol (GP), 20. toluène, 21. diméthylhexane + éthylpropylsulfure (GP), 22. n-pentanol, 23. biméthylcyclohexane (GP), 24. hexanal, 25. éthylbutanoate (GP), 26. propylpropanoate + 4méthyl-penten-2-al-1, 27. n.i. (GP), 28. butylacétate + octane + tétrachloroéthylène (GP), 29. pyrrole, 30 3-méthylhexane-2-one, 31. produit soufré $\mathrm{M}=118$ (G.), 32. cétone insaturée (GP), 33. furfural, 34. 5-méthyl-2-hexanone, 35. méthylhexanal, 36. éthylbenzène, 37. xylène, 38. acétate d'isoamyle (G.), 39. hexanol (GP), 40. 2-heptanone, 41. styrène, 42. heptanal, 43. méthional, 44. 2-heptanol + 5-méthyl-hex-trans-2-enal, 45. méthyloctane (GP), 46. 2-éthylpyrazine (GP), 47. acétylfurane, 48. furfurol, 49. propylbenzène (GP), 50. carbonyle monoinsaturé $\mathrm{M}=126$, 51. méthylheptanone-2, 51'. I-acétylpyrrole (G.), 52. benzaldéhyde, 53. carbonyle monoinsaturé $M=126$, 54. heptanol (GP), 55. 2-octanone (G), 56. méthylheptanal, 57. éthylhexanoate + triméthylpyridine, 58. pentylfurane (GP), 59. octanal + triméthylpyrazine, 60 . decane + dichlorobenzène, 61. propionylfurane (GP), 62. 2-acétylpyrrole, 63, phénylacétaldẻhyde, 64. acétophénone, 65. 2-éthyl-3.6diméthylpyrazine, 66. octanol, 67. alcool benzylique, 68. 2-nonanone, 69. nonanal + phénol, 70. 2-nonanol, 71. méthylnonénone, 72. n.i., 73. méthyléthylpyrrole, 74. phénylpropanone-2, 75. phényléthanol, 76. nonénal + 2-éthyl-3.5.6.-triméthylpyrazine, 77. phényl-2-propenal (GP), 78. phénylpropanone-1 (G.), 79. crésol, 80. nonanol (GP), 81. trichlorobenzène, 82. phénylpentane (G), 83. 2-décanone, 84. naphtalène + éthyldécanoate, 85. décanal, 86. dodecane, 87. dérivé du pyrrole, 88. triéthylpyrazine, 89. benzothiazole, 90. $\gamma$-lactone (?), 91. décanol, 92. 2-phényl2-butenal-1, 93, 2-undecanone, 94. méthylnaphtalène (GP), 95. déca-2,4-diénal, 96. tridecane (G), 97. $\gamma$-lactone (GP), 98. 4-méthyl-2-phényl-penten-2-al-1, 99. indole.

\section{III. - RESULTATS ET DISCUSSION}

Les résultats ont été regroupés dans une série de neuf tableaux à sept colonnes se rapportant chacun à une classe chimique définie. La première colonne marquée $F$ concerne les produits volatils identifiés dans le Gruyère de Comté utilisé. Les secondes et troisièmes colonnes (F.G.) et (F + P.G.) se rapportent aux produits volatils extraits respectivement du fromage et des pâtes gratinées. La quatrième colonne marquée $P$ concerne les produits volatils extraits des pâtes cuites. La cinquième colonne et la sixième se rapportent aux produits identifiés dans les vapeurs dégagées pendant la cuisson au four du fromage seul et du fromage associé aux pâtes. La dernière colonne enfin montre quels sont les produits volatils qui ont pu être identifiés dans le fromage gratiné par une méthode de " head space » associée au piégeage sur Porapak. 
TABLEAU 1. - Aldéhydes

\begin{tabular}{|c|c|c|c|c|c|c|c|c|}
\hline & $\begin{array}{c}\text { pic } n^{\circ} \\
\text { (fig. 2) }\end{array}$ & F seul & F.G. & $(F+P) G$ & Ps & V.F.G. & $V(F+P) G$ & Jennings G \\
\hline Ethanal & 1 & + & + & + & & + & + & \\
\hline 2-méthylpropanal & 3 & & + & + & + & & + & + \\
\hline 2-méthylbutanal & 8 & + & + & + & & + & + & + \\
\hline $\begin{array}{l}\text { 3-méthylbutanal } \\
\text { Pentanal }\end{array}$ & 7 & + & + & + & $\begin{array}{l}+ \\
+\end{array}$ & + & $\begin{array}{l}+ \\
+\end{array}$ & + \\
\hline Hexanal & 24 & + & + & + & + & + & + & + \\
\hline Méthylhexanal & 35 & & + & + & + & & & \\
\hline Heptanal & 42 & + & + & + & + & + & + & + \\
\hline Méthylheptanal & 56 & & + & + & & & & \\
\hline Octanal & 59 & & + & + & & + & + & \\
\hline Nonanal & 69 & $+?$ & + & + & + & + & + & + \\
\hline Décanal & 85 & $+?$ & + & + & & + & + & \\
\hline 2-méthyl-2-buten-al-1 & 15 & & + & + & & & + & \\
\hline 4-méthyl-2-penten-al-1 & 26 & sous réserve & + & + & & & + & + \\
\hline 5-méthyl-2-hexen-al-1 & 44 & & + & + & & & + & + \\
\hline 2-nonenal & 76 & & + & + & + & & + & \\
\hline Benzaldéhyde & 52 & + & + & + & + & + & + & + \\
\hline 2-phényl-éthanal & 63 & + & + & + & & + & + & + \\
\hline 2-phényl-2-propenal & 77 & $\begin{array}{c}\text { sous réserve } \\
\text { traces }\end{array}$ & & + & & & & \\
\hline Phényl-2-buten-al-1 & 92 & + & + & + & & + & & \\
\hline 4-methyl-2-phényl-2-penten-al & 98 & & + & + & & & & \\
\hline Déca-2,4-diénal & 95 & & + & + & + & + & + & \\
\hline
\end{tabular}


Il est possible de se faire une idée des quantités relatives de chacun des produits identifiés dans le fromage gratiné seul ou avec des pâtes (colonnes 2 et 3 ) en comparant les chromatogrammes reproduits à la figure 2 . Ceux-ci, obtenus dans les mêmes conditions à partir d'un volume d'extrait correspondant à un même poids de fromages comportent un nombre important de pics qui ont été repérés par une numérotation identique dans les deux cas.

L'examen de la colonne 4 consacrée aux pâtes montre que ces dernières ne jouent pratiquement pas de rôle comme précurseur de produits volatils mais que leur action n'est pas négligeable du fait de leur forte teneur en humidité qui a pour conséquence de limiter la température à laquelle est porté le fromage lorsqu'il est gratiné avec des pâtes.

Ceci explique que le chromatogramme obtenu à partir du fromage gratiné seul présente un profil nettement plus chargé que celui obtenu à partir du fromage gratiné avec les pâtes.

L'examen du tableau 1 consacré aux aldéhydes montre que s'il existe toute une série d'aldéhydes dans le fromage de Comté, celles-ci ne sont pour la plupart présentes qu'à l'état de traces. La cuisson par contre est génératrice de grandes quantités d'aldéhydes aliphatiques à courte chaîne (y compris de méthanal qui a pu être mis en évidence en larges quantités dans les vapeurs émises, au moyen de la chromatographie en couche mince) et en particulier d'aldéhydes ramifiés, résultant de dégradation de Strecker, que l'on a mis en évidence aussi bien dans le produit final que dans les vapeurs. Le chauffage joue également un rôle prépondérant dans la formation des aldéhydes aromatiques à tel point que benzaldéhyde et phénylacétaldéhyde sont parmi les composés volatils quantitativement majeurs des produits gratinés. Le 2-4 décadiénal n'a pas pu être mis en évidence dans le fromage mais il est présent dans les produits cuisinés.

Ces résultats sont en accord avec ceux de Nakaniski et Itoh [12] qui ont observé la formation d'éthanal, propanal, 2-méthyl propanal, 3-méthyl-butanal et 2-méthyl-butanal lors de l'autoclavage de caséine en solution à $140^{\circ} \mathrm{C}$ pendant $60 \mathrm{mn}$ et avec ceux de Kato et al. [8] qui en plus de ces composés observent la formation de butanol, de benzaldéhyde et d'acétophénone par chauffage de caséine à sec à $250^{\circ} \mathrm{C}$ pendant $1 \mathrm{~h}$. Plus récemment, Quist et Von Sydow [14] observent également la formation d'aldéhydes ramifiés à courte chaîne lors du chauffage de coprécipité en présence soit d'eau, soit de matière grasse non butyrique et d'amidon.

Le gratin se singularise par rapport aux diverses protéines de lait chauffées par la présence d'aldéhydes aromatiques présentes en quantités d'autant plus importantes que le chauffage a été plus sévère (pics 92 et 98). Kinlin et al. [9] ont signalé la formation de tels aldéhydes aromatiques au cours de grillage des noisettes. 
TABLEAU 2. - Cétones

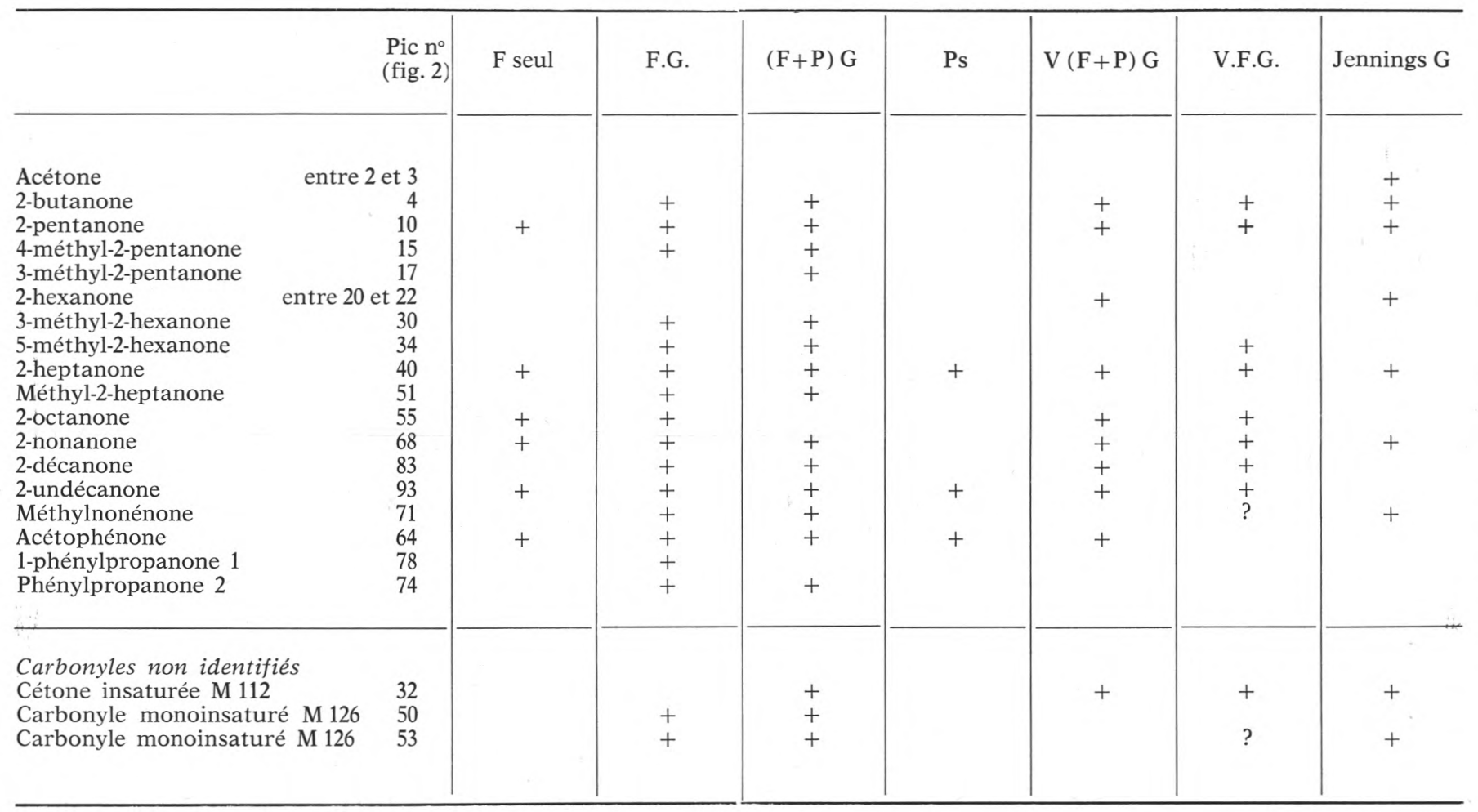


TABLEAU 3. - Alcools

\begin{tabular}{|c|c|c|c|c|c|c|c|c|}
\hline & $\begin{array}{c}\text { Pic no } \\
\text { (fig. 2) }\end{array}$ & F seul & F.G. & $(F+P) G$ & Ps & $V(F+P) G$ & V.F.G. & Jennings $\mathrm{G}$ \\
\hline $\begin{array}{l}\text { Méthanol } \\
\text { Ethanol } \\
\text { Propanol } \\
\text { 2-méthyl-1-propanol } \\
\text { Butanol } \\
\text { 2-méthylbutanol } \\
\text { 3-méthylbutanol } \\
\text { Pentanol } \\
\text { Hexanol } \\
\text { Heptanol } \\
\text { Octanol } \\
\text { Nonanol } \\
\text { Décanol } \\
\text { Benzylalcool } \\
\text { Phényléthanol } \\
\text { 2-butanol } \\
\text { 2-pentanol } \\
\text { 2-heptanol } \\
\text { 2-nonanol } \\
\text { Oct-1-en-ol-3 } \\
\text { Acétoïne } \\
\text { 31-45-59...87-102 }\end{array}$ & $\begin{array}{r}2 \\
\text { entre } 2 \text { et } 3 \\
4 \\
\text { niv. } 7 \\
10 \\
19 \\
18 \\
22 \\
39 \\
54 \\
66 \\
80 \\
91 \\
67 \\
75 \\
5 \\
13 \\
44 \\
70 \\
\text { niv. } 57 \\
14 \\
27\end{array}$ & $\begin{array}{l}+ \\
+ \\
+ \\
+ \\
+ \\
+ \\
+ \\
+ \\
+ \\
+ \\
+ \\
+ \\
+ \\
+ \\
+ \\
+ \\
+ \\
+ \\
+\end{array}$ & $\begin{array}{l}+ \\
+ \\
+ \\
+ \\
+ \\
\\
+ \\
+ \\
+ \\
+ \\
+ \\
+ \\
+ \\
+ \\
+ \\
+\end{array}$ & $\begin{array}{l}+ \\
+ \\
+ \\
+ \\
+ \\
+ \\
+ \\
+ \\
+ \\
+ \\
+ \\
+ \\
+ \\
+ \\
+ \\
+ \\
+ \\
+ \\
+\end{array}$ & $\begin{array}{l} \\
+ \\
+ \\
+ \\
+ \\
+ \\
+\end{array}$ & $\begin{array}{l}+ \\
+ \\
+ \\
+ \\
+ \\
+ \\
+ \\
+ \\
+ \\
+ \\
+ \\
+ \\
+ \\
+ \\
+\end{array}$ & $\begin{array}{l}+ \\
+ \\
+ \\
+ \\
+ \\
+ \\
+ \\
+ \\
+ \\
+ \\
+ \\
+\end{array}$ & $\begin{array}{l}+ \\
+ \\
+ \\
+ \\
+\end{array}$ \\
\hline
\end{tabular}


TABLEAU 4. - Esters

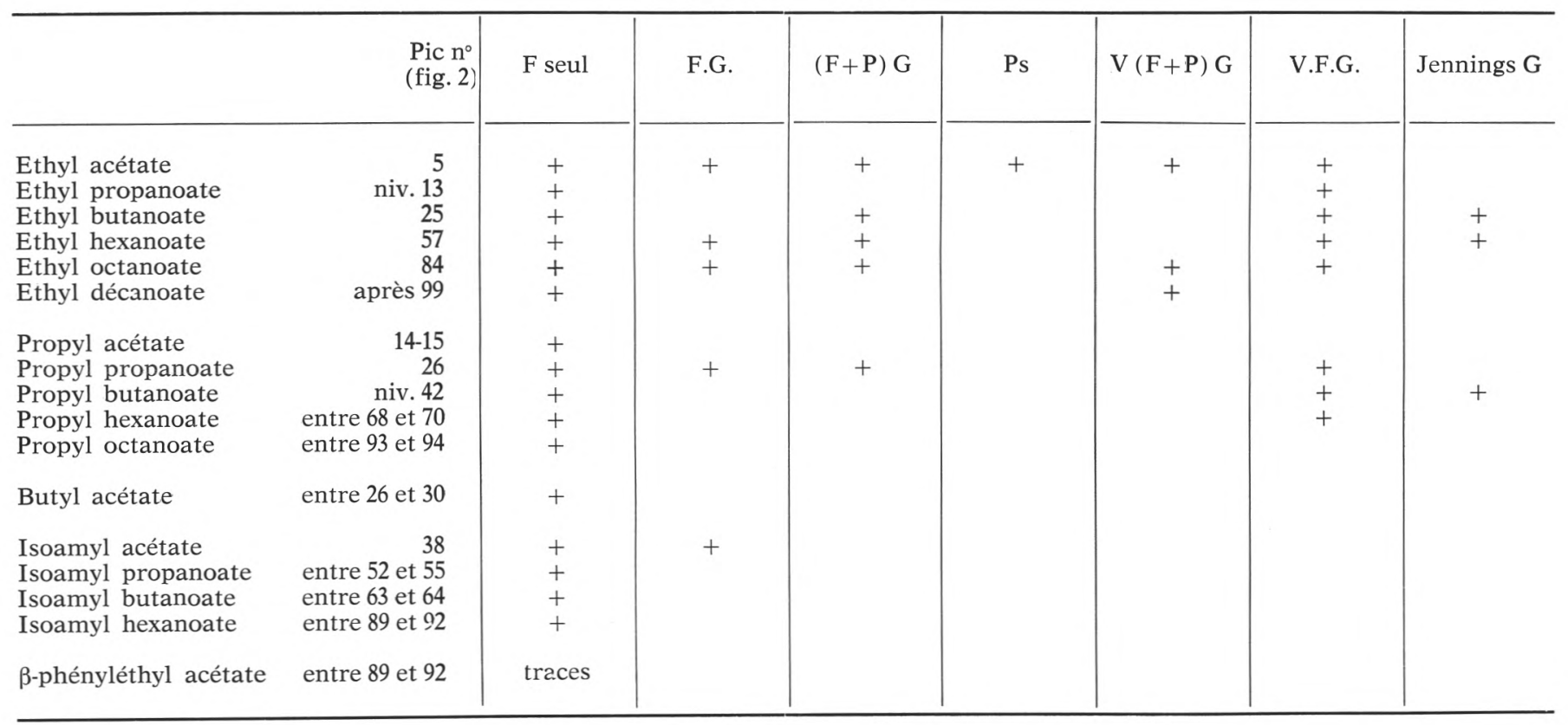


Le tableau 2 montre que le chauffage a pour résultat d'augmenter le nombre et la quantité des cétones présentes, ce qui est en accord avec le mécanisme connu de formation des cétones à partir des $\beta$-céto-esters par chauffage en présence d'eau. On notera en particulier la formation de 2-décanone (pic 83) en assez forte quantité lorsque l'intensité du chauffage augmente et la présence de phénylcétones. Il faut noter que l'acétophénone qui est un composé ordinairement présent dans les fromages à croûte lavée a pu être mis en évidence dans le Comté même avant chauffage. Il faut probablement y voir là l'influence de la morge puisque ce composé n'a pas été signalé dans les travaux consacrés aux fromages type Emmental [10].

Les alcools et plus particulièrement les alcools primaires à courte chaîne représentent une part importante des produits volatils isolés du fromage. Parmi les alcools secondaires les plus importants sont le 2-butanol et l'acétoïne. L'intensité du chauffage semble avoir une influence sur la formation de 2-heptanol.

La présence du oct-1-en-ol-3 dans les pâtes est à rapprocher de celle de la benzaldéhyde, du 2,4-décadiénal et d'aldéhydes aliphatiques, composants dénotant l'existence d'un phénomène oxydatif.

Les esters sont particulièrement nombreux dans le Comté (tab. 4). Un certain nombre d'esters propyliques ont pu être mis en évidence alors que leur absence avait été soulignée par Langler [10] dans le fromage type Emmental. La plupart des esters semblent disparaître à la cuisson.

Le chauffage est générateur de nombreux hydrocarbures en particulier d'hydrocarbures ramifiés qui sont présents aussi bien dans le produit final que dans les vapeurs. Les hydrocarbures benzéniques sont des composants normaux du fromage (tab. 5).

Le fromage contient normalement un certain nombre de composés soufrés (tab. 6). Certains d'entre eux très volatils ou peu stables n'y apparaissent pas. Seuls y sont mentionnés ceux qui ont pu être identifiés par spectrométrie de masse. On peut toutefois apprécier la quantité de ces produits très volatils initialement présents dans le fromage en se rapportant d'une part au tableau 6 bis où figurent les résultats obtenus par dosage colorimétrique et d'autre part à la figure 3 qui reproduit les chromatogrammes obtenus en utilisant un détecteur spécifique du soufre et une technique de " head space ", et sur lesquels les pics n'ont été identifiés que par leur temps de rétention. On y voit figurer parmi les plus volatils l'hydrogène sulfuré, le méthanethiol et le sulfure de méthyle ainsi que certaines substances non identifiées mais qui pourraient correspondre aux composés identifiés par spectrométrie de masse (acétate de méthanethiol, diéthyldisulfure, etc.).

La cuisson provoque une formation abondante d'hydrogène sulfuré, que l'on retrouve pour la plus grapde part dans les vapeurs, 
TABLEAU 5. - Hydrocarbures

\begin{tabular}{|c|c|c|c|c|c|c|c|c|}
\hline & $\begin{array}{c}\text { Pic no } \\
\text { (fig. 2) }\end{array}$ & F seul & F.G. & $(F+P) G$ & Ps & $V(F+P) G$ & V.F.G. & Jennings G \\
\hline $\begin{array}{l}\text { 2,3-diméthylbutane } \\
\text { 2-méthylpentane } \\
\text { Hexane } \\
\text { Ethylpentane } \\
\text { Diméthylhexane } \\
\text { Octane } \\
\text { Méthyloctane } \\
\text { Décane } \\
\text { Undécane } \\
\text { Dodécane } \\
\text { Tridécane } \\
\text { Diméthylcyclohexane } \\
\text { Benzène } \\
\text { Toluène } \\
\text { Ethylbenzène } \\
\text { Xylène } \\
\text { Propylbenzène } \\
\text { Phénylpentane } \\
\text { Styrène } \\
\text { Naphtalène } \\
\text { Méthylnaphtalène }\end{array}$ & $\begin{array}{c}(4) \\
(4) \\
(5) \\
(13) \\
21 \\
28 \\
45 \\
60 \\
\text { entre } 69 \text { et } 71 \\
86 \\
96 \\
23 \\
9 \\
20 \\
36 \\
37 \\
49 \\
82 \\
41 \\
84 \\
94\end{array}$ & $\begin{array}{c}\text { traces } \\
+ \\
\\
\\
\\
+ \\
+ \\
+ \\
+ \\
\\
\\
+ \\
+ \\
+ \\
+ \\
+ \\
\text { souserse } \\
+ \\
+ \\
+\end{array}$ & $\begin{array}{l}+ \\
+ \\
+ \\
+ \\
+ \\
+ \\
+ \\
+ \\
+ \\
+ \\
+ \\
+ \\
+ \\
+ \\
+ \\
+ \\
+\end{array}$ & $\begin{array}{l}+ \\
+ \\
+ \\
+ \\
+ \\
+ \\
+ \\
+ \\
+ \\
+ \\
+ \\
+ \\
+ \\
+ \\
+ \\
+ \\
+ \\
+ \\
+\end{array}$ & $\begin{array}{l}+ \\
+ \\
+ \\
+\end{array}$ & $\begin{array}{l}+ \\
+ \\
+ \\
+ \\
+ \\
\\
+ \\
+ \\
\\
+ \\
+ \\
+ \\
+ \\
+ \\
+ \\
+ \\
+ \\
+\end{array}$ & $\begin{array}{l}+ \\
+ \\
+ \\
+ \\
+ \\
+ \\
+ \\
+\end{array}$ & $\begin{array}{l}+ \\
+ \\
+\end{array}$ \\
\hline
\end{tabular}


TABLEAU 6. - Composés soufrés

\begin{tabular}{|c|c|c|c|c|c|c|c|c|}
\hline & $\begin{array}{c}\text { Pic no } \\
\text { (fig. 2) }\end{array}$ & F seul & F.G. & $(F+P) G$ & Ps & $V(F+P) G$ & V.F.G. & Jennings $\mathrm{G}$ \\
\hline Diméthyldisulfure & $(16)$ & + & + & + & & + & & + \\
\hline Acétate de Méthanethiol & niv. 10 & + & & + & & & & \\
\hline Ethylpropylsulfure & 21 & & & & & & & \\
\hline Diéthyldisulfure & niv. 46 & + & & & & & & \\
\hline Méthional & 43 & & + & + & & & & + \\
\hline Méthylthiophène & 20 et 26 & + & & & & & & \\
\hline Benzothiazole & 89 & + & + & + & & & + & \\
\hline Prod. $M=113$ avec maj. 8 & 31 & & + & & & & & \\
\hline
\end{tabular}



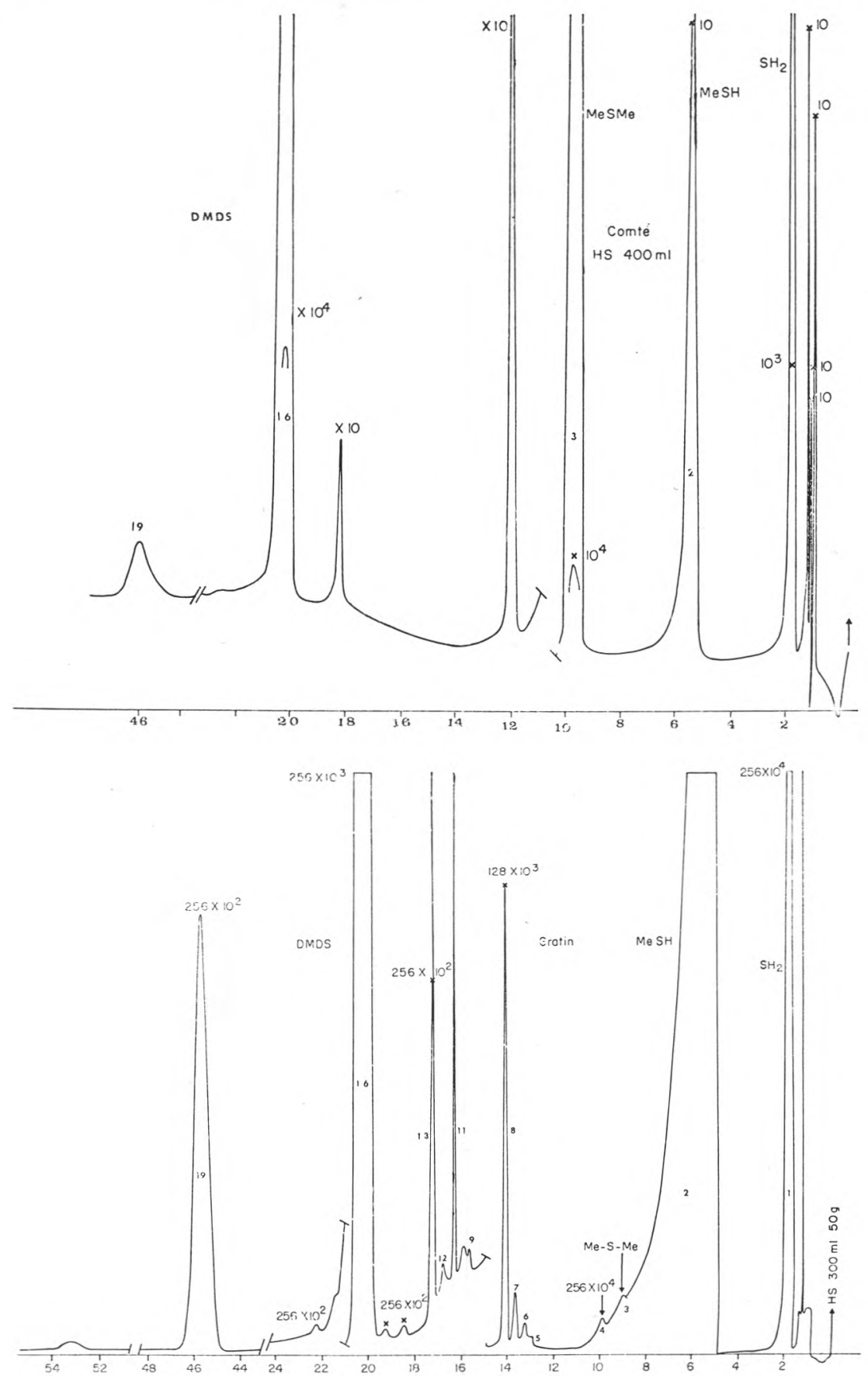

fig. 3

(a) Composés soufrés présents dans le fromage de Comté $(\mathrm{F})$.

(b) Composés soufrés présents après gratinage du fromage seul (G). Colonne : $6,3 \mathrm{~m} \times 3 \mathrm{~mm}$ - verre 5 p. 100 Igepal CO 630 sur chrom G AW. DMCS $8010020 \rightarrow 120^{\circ} \mathrm{C} 10^{\circ} \mathrm{C} / \mathrm{mn}-$ Head Space $400 \mathrm{ml}$.

1. hydrogène sulfuré, 2. méthanethiol, 3. diméthylsulfure, 13. thiophène (G), 16. diméthyldisulfure, 19. 3,5-diméthyl-:,2,4-trithiolane. 


\section{TABLEAU 6 bis}

Dosage des produits soufrés présents dans le fromage et formés au cours du chauffage

\begin{tabular}{|c|c|c|c|}
\hline & $\mathrm{H}_{2} \mathrm{~S}$ & Thiols $^{1}$ & Disulfures $^{2}$ \\
\hline Fromage & $\begin{array}{ll}\text { d.o. } & 0 \\
\text { p.p.m. } 0\end{array}$ & $\begin{array}{ll}\text { d.o. } & 0,018 \\
\text { p.p.m. } & 0,026\end{array}$ & $\begin{array}{ll}\text { d.o. } & 0,012 \\
\text { p.p.m. } & 0,014\end{array}$ \\
\hline Vapeurs émises & $\begin{array}{ll}\text { d.o. } & 0,610 \times 10 \\
\text { p.p.m. } & 1,80\end{array}$ & $\begin{array}{ll}\text { d.o. } & 1,200 \\
\text { p.p.m. } & 1,2\end{array}$ & $\begin{array}{ll}\text { d.o. } & 0,058 \\
\text { p.p.m. } & 0,075\end{array}$ \\
\hline Gratin & $\begin{array}{ll}\text { d.o. } & 0,255 \\
\text { p.p.m. } & 0,07\end{array}$ & $\begin{array}{l}\text { d.o. } \quad 1,000 \\
\text { p.p.m. } 1\end{array}$ & $\begin{array}{ll}\text { d.o. } & 0,027 \\
\text { p.p.m. } & 0,035\end{array}$ \\
\hline
\end{tabular}

(1) Exprimé en méthanethiol.

(2) Exprimés en disulfure de méthyle.

de méthanethiol et de disulfures. On notera sur la figure 3 (b) l'apparition de plusieurs composés dont le thiophène (pic 13) ainsi que l'augmentation des quantités de 3,5-diméthyl-1,2,4-trithiolane (pic 19) qui existait déjà dans le fromage. Ce composé susceptible de se former à partir d'acétaldéhyde et d'hydrogène sulfuré a déjà été signalé dans un certain nombre de produits chauffés carnés $[2,16]$ ou végétaux [3] ainsi que dans le coprécipité [14].

Un certain nombre de pyrazines sont présentes à l'état de traces dans le fromage (tab. 7). Ces résultats recoupent ceux de Sloot et Hofman [15] qui ont récemment mis en évidence ces pyrazines dans l'Emmental. La cuisson augmente notablement les quantités de ces substances dont certaines sont présentes dans les vapeurs, et fait également apparaître plusieurs dérivés de la pyridine. Le chauffage fait également apparaître des dérivés du furane et du pyrrole qui peu volatils se retrouvent surtout dans le produit consommable.

\section{CONCLUSION}

Parmi tous les produits volatils répertoriés dans cette étude seul un nombre plus restreint est présent dans les vapeurs et dans le condensat obtenu par la méthode de Jennings, condensat qui dégage pourtant une odeur caractéristique de gratin. On peut donc en conclure que sur le plan de l'odeur seuls les composés communs à la fois au gratin et aux vapeurs sont à considérer comme importants. Ceci conduit à souligner le rôle des aldéhydes et des produits 
TABLEAU 7. - Composés azotés

\begin{tabular}{|c|c|c|c|c|c|c|c|c|}
\hline & $\begin{array}{c}\text { Pic } n^{\circ} \\
\text { (fig. 2) }\end{array}$ & F seul & F.G. & $(F+P) G$ & Ps & $V(F+P) G$ & V.F.G. & Jennings G \\
\hline $\begin{array}{l}\text { Méthylpyrazine } \\
\text { 2,3-diméthylpyrazine } \\
\text { 2,5-diméthylpyrazine } \\
\text { Triméthylpyrazine } \\
\text { 2-éthyl-3,6-diméthylpyrazine } \\
\text { Tétraméthylpyrazine } \\
\text { 2-éthyl-3,5,6,-triméthylpyrazine } \\
\text { Triéthylpyrazine } \\
\text { Indole } \\
\text { Pyridine } \\
\text { 2-méthylpyridine } \\
\text { 4-méthylpyridine } \\
\text { 3-éthylpyridine } \\
\text { Diméthylpyridine } \\
\text { Triméthylpyridine } \\
\text { 2-méthyl-5-éthylpyridine } \\
\text { Butylpyridine } \\
\text { 4-pentylpyridine }\end{array}$ & $\begin{array}{r}46 \\
59 \\
65 \\
7 i v .67 \\
76 \\
88 \\
99 \\
\end{array}$ & $\begin{array}{l}+ \\
+ \\
+ \\
+ \\
+ \\
+ \\
+\end{array}$ & $\begin{array}{l}+ \\
+ \\
+ \\
+ \\
+ \\
+ \\
+ \\
+ \\
+ \\
+ \\
+ \\
+ \\
+ \\
+ \\
+ \\
+ \\
+\end{array}$ & $\begin{array}{l}+ \\
+ \\
+ \\
+ \\
+ \\
+ \\
+ \\
+ \\
+ \\
+ \\
+ \\
+ \\
+ \\
+ \\
+ \\
+ \\
+ \\
+ \\
+\end{array}$ & + & + & $\begin{array}{l}+ \\
+ \\
+ \\
+ \\
+\end{array}$ & + \\
\hline
\end{tabular}


TABLEAU 8. - Dérivés du Furane et du Pyrrole

\begin{tabular}{|c|c|c|c|c|c|c|c|c|}
\hline & $\begin{array}{l}\text { Pic } n^{\circ} \\
\text { (fig. 2) }\end{array}$ & F seul & F.G. & $(F+P) G$ & Ps & $V(F+P) G$ & V.F.G. & Jennings G \\
\hline \multicolumn{9}{|l|}{ Dérivés du furane } \\
\hline Tétrahydrofurane & (6) & & & + & & & & \\
\hline Ethylfurane & (12) & & & + & & & & \\
\hline Pentylfurane & $(58)$ & & & + & & & & \\
\hline Acétylfurane & $(47)$ & & + & + & & & + & + \\
\hline $\begin{array}{l}\text { Propionylfurane } \\
\text { Furfural }\end{array}$ & $\begin{array}{l}(61) \\
33\end{array}$ & & + & $\begin{array}{l}+ \\
+\end{array}$ & + & & & \\
\hline \multicolumn{9}{|l|}{ Dérivés du pyrrole } \\
\hline Pyrrole & 29, & & + & + & & + & + & \\
\hline 1-acétylpyrrole & $51^{\prime}$ & & + & & & & + & \\
\hline $\begin{array}{l}\text { 2-acétylpyrrole } \\
\text { Méthyléthylpyrrole }\end{array}$ & $\begin{array}{l}62 \\
73\end{array}$ & & + & $\begin{array}{l}+ \\
+\end{array}$ & & & t & \\
\hline Non identifié & 87 & & + & + & & & & \\
\hline
\end{tabular}


TABLEAU 9. - Composés chlorés et composés divers

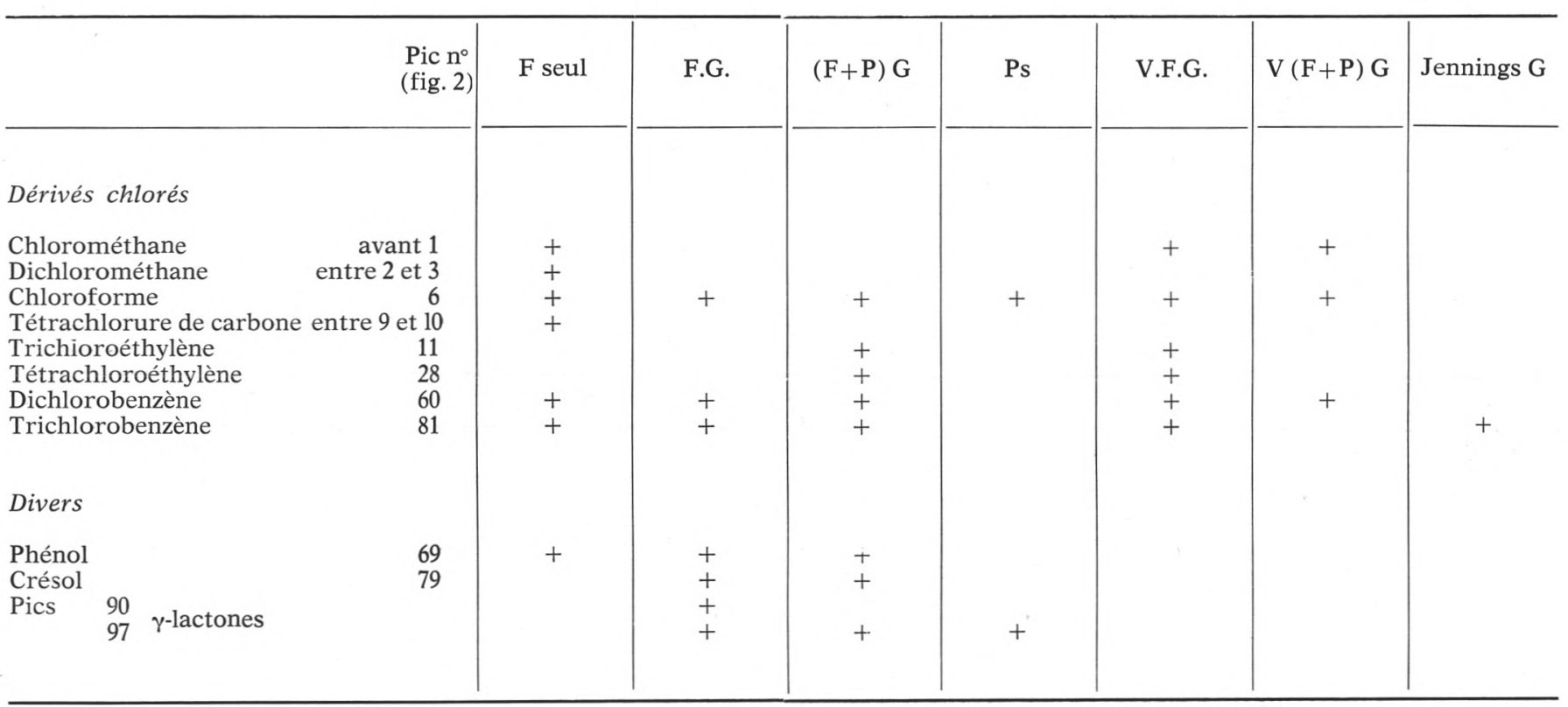


soufrés qui sont d'autant plus importants que leur seuil de perception est particulièrement bas. Les classes de composés à seuil de perception assez important comme les alcools ou les hydrocarbures doivent pouvoir être considérés comme moins importants.

Sur le plan de l'arôme il est certain qu'un plus grand nombre de classes chimiques doivent être prises en considération. Aux composés impliqués dans l'odeur il faut ajouter des composés qui n'étaient pas assez volatils puur apparaître dans les vapeurs mais dont le pouvoir aromatique est connu (pyrazines, par exemple). On peut remarquer que dans l'ensemble ce sont surtout des produits qui proviennent de la dégradation des protéines qui apparaissent comme importants, la matière grasse joue un rôle beaucoup moins net.

\section{Rés u m é}

Les composés volatils formés au cours du gratinage de fromage de Comté utilisé seul ou en association avec des pâtes ont été identifiés par spectrométrie de masse. On note, en particulier dans les vapeurs dégagées au cours de la cuisson, une apparition d'aldéhydes aliphatiques ramifiés à courte chaîne et de produits soufrés.

\section{S u m m a r y}

The volatile compounds which arise from the cooking of Gruyère cheese have been studied by GC-MS- Branched short chain aldehyde and sulfur compounds seems to be most important. Numerous other compounds have been identified.

Reçu pour publication en septembre 1975.

\section{Références}

[1] Badings (H. T.), Wassink (J. G.) (1963). - Neth. Milk Dairy J., 17, 132.

[2] Bunkman (H. W.), Copier (H.), de Leuw (J. J. M.), TJan (S. B.) (1972). J. Agric. Food Chem., 20, 177.

[3] Buttery (R. G.), Seifert (R. M.), Ling (Louisa C.) (1975). - J. Agric. Food Chem., 23, 516.

[4] Dhont (J. H.), DiJkman (G. J. C.) (1967). - Analyst., 92, 431. 
[5] Dumont (J. P.), Roger (Sylviane), Adda (J.) (1974). - Le Lait, 31, 531--532.

[6] Forss (D. A.), Jacobsen (Valérie M.), Ramshaw (E. H.) (1967). - J. Agric. Food Chem., 15 (6).

[7] Jennings (W. G.), Wohkeb (R.), Lewis (M. J.) (1972). - J. Fd. Science, 37, 69.

[8] Kato (H.), Hayase (F.), Fujimaki (M.) (1972). - Agr. Biol. Chem., 36, 951.

[9] Kinlin (T. E.), Muralidhara (R.), Pittet (A. O.), Sanderson (A.), Wabradt (P. J.) (1972). - J. Agr. Food Cherr., 20, 1021.

[10] LangleR (J. E.) (1966). - Thesis, Corvallis, Oregon State University.

[11] Mc Lay (R.) (1967). - J. Sci. Fd. Agric., 18, 12.

[12] Nakanishi (T.), Iтон (T.) (1967). - Agr. Biol. Chem., 31, 1066.

[13] Palmer (J. K.) (1973). - J. Agr. Food Chem., 21, 923.

[14] Qvist (I. H.), Von Sydow (E. C. F.) (1974). - J. Agr. Food Chem., 22, 1077.

[15] Sloot (D.), Hofman (J. H.) (1975). - J. Agr. Food Chem., 23, 2.

[16] Wilson (R. A.), Mussinan (C. J.), Katz (I.), Sanderson (A.) (1973). - J. Agr. Food Chem., 21, 873. 